\section{Role of ANA testing in the classification of patients with systemic lupus erythematosus}

The recent publication ${ }^{12}$ of the European League Against Rheumatism (EULAR)/American College of Rheumatology (ACR) criteria for the classification for systemic lupus erythematosus (SLE) provides an important reason to continue the dialogue on testing for antinuclear antibodies (ANA) that has appeared in Annals of the Rheumatic Diseases (ARD). Following our article on the variability of immunofluorescence assays (IFA) for testing ANA in $\mathrm{SLE}^{3}{ }^{3}$ the journal published a series of very interesting and informative letters addressing our findings and reporting on other experience in ANA testing. ${ }^{4-12}$ These letters have been important in alerting the field to the challenges in ANA testing and the variety of assay approaches, including solid phase assays (SPA), that are currently available for determining the serological status of patients.

While our focus was the clinical trial setting, our results are also relevant to the new classification criteria. ${ }^{12}$ These criteria differ from previous classification systems in the positioning of a positive ANA. Whereas a positive ANA represented one criterion in the ACR and Systemic Lupus International Collaborating Clinics criteria, ${ }^{13}{ }^{14}$ in the new EULAR/ACR criteria, a positive ANA is necessary for classification; if this criterion is met, other manifestations receive varying numbers of points to determine classification. Such a positioning implies that SLE is always ANA positive, that a negative ANA can effectively rule out SLE and that conventional ANA testing can reliably make this determination. We do not believe that these contentions are supported by the available data and have previously commented on this issue. $^{15}$

In the initial version of the EULAR/ACR criteria, an IFA was specified as the assay for ANA assessment based on a comprehensive review of the literature ${ }^{16}$ indicating that frequency of ANA positivity in SLE is very high $(97.8 \%$ with a CI of $96.8 \%$ to 98.5\%). Much higher values for ANA negativity (4.9\%-22.3\%) in SLE with different kits were reported in our study in ARD. A subsequent study of patients enrolled in a trial of anti-IL-6 showed ANA negativity ranging from $0.6 \%$ to $27.6 \%$, depending on the kit. ${ }^{17}$ The frequency of ANA negativity was also high in the phase 2 belimumab trial. ${ }^{18}$

In the final version of the criteria just published, however, the requirement for a positive ANA by IFA was changed to allow, 'a solid phase ANA screening immunoassay with at least equivalent performance...' to the IFA as an alternative. The change in assays for ANA assessment is very reasonable given the limited access to the IFA in many practice settings; indeed, availability of the IFA may dwindle further since testing laboratories have a preference for high throughput and automated systems and, pending the refinement of digitised systems for computer reading of IFA results, SPA are likely to be commonly used. ${ }^{19}$

Differences between IFA and SPA are many and, just as IFA kits can produce variable results, so too can SPA. Furthermore, an individual serum can be positive in the IFA and negative in an SPA and vice versa. This situation is not surprising given the construction of the SPA (eg, use of a limited number of cloned or purified proteins) as well as the uncertain representation of certain autoantigens in the IFA (eg, Ro). ${ }^{19}$ To us, the key issue in determining assays for ANA testing for classification does not relate to the relative merits of the IFA versus SPA. Rather, the issue relates to assay validation and the manner in which equivalent performance is determined. It is our opinion that including ANA in the classification criteria without designating the sensitivity and specificity of available assays (either IFA or SPA) in the context of the intended use could lead to significant patient misclassification.

ANA assays are, in general, validated for testing of autoantibody-related rheumatic diseases (AARD), such as SLE, progressive systemic sclerosis and Sjogren's syndrome. While these conditions are frequently ANA positive, determination of specific autoantibodies, such as anti-Ro, anti-La or anti-SCL70, is usually much more informative than the ANA. Furthermore, whereas validation of particular kits may involve testing of large numbers of sera from patients with different conditions, the nature of the patient populations studied may impact on the results since disease duration and prior treatment can potentially affect ANA positivity. We believe that our findings on ANA negativity in SLE ${ }^{317}$ as well as the belimumab study, ${ }^{18}$ are important and may represent the natural history of SLE (or effects of treatment) as it evolves into a stage that can be termed post-autoimmunity.

Given the importance of ANA testing in the new classification criteria, we believe that it is essential to determine the actual frequency of ANA positivity in patients with SLE assessing kits currently used by testing laboratories. Optimally, such validation should be performed with sera from patients with SLE at various stages of the disease and involve direct comparisons between different kits with the same set of sera to establish equivalent performance. For validation purposes, reliance on published results may not be adequate in view of potential differences in the patient populations in different studies. Furthermore, we believe that it is important to have a wide representation of racial and ethnic groups in view of data that certain ANA responses occur more commonly in populations such as African-American or Afro-Caribbean. ${ }^{20}$

The new criteria for SLE are a major innovation and we applaud the Steering Committee for organising and conducting such a detailed, thoughtful and evidence-based international enterprise. While we agree that a positive ANA should be an important and possibly essential criterion, unfortunately, the field lacks a gold standard for ANA determination with either the IFA or SPA. In fact, we do not know how often SLE is truly ANA negative since it depends on the assay. In this regard, it is possible that one assay may not suffice for this purpose and that a combination of different IFA and SPA kits may work best. ${ }^{4791221}$

SLE is a complicated and heterogeneous disease whose correct classification is critical for patient management and scientific study. The new EULAR/ACR criteria provide a valuable framework. Further research is, therefore, critical at this time to validate assays not for the detection of ANA in AARD but rather for the specific setting of SLE classification.

\section{David S Pisetsky, ${ }^{01,2}$ Diane M Spencer, ${ }^{1,2}$ Brad Rovin, $^{3}$ Peter E Lipsky ${ }^{4}$ \\ ${ }^{1}$ Medicine, Duke University Medical Center, Durham, North Carolina, USA ${ }^{2}$ Veterans Administration Medical Center, Medical Research Service, Durham, North Carolina, USA \\ ${ }^{3}$ Medicine, Ohio State University, Columbus, Ohio, USA \\ ${ }^{4}$ RILITE Institute, Charlottesville, Virginia, USA}

Correspondence to Dr David S Pisetsky, Medicine, Duke University Medical Center, Durham, NC 27705, USA; david.pisetsky@duke.edu

Handling editor Josef S Smolen

Contributors All the authors contributed to the development of this correspondence.

Funding The authors have not declared a specific grant for this research from any funding agency in the public, commercial or not-for-profit sectors.

Competing interests None declared.

Patient consent for publication Not required. 
Provenance and peer review Not commissioned; internally peer reviewed.

(C) Author(s) (or their employer(s)) 2019. No commercial re-use. See rights and permissions. Published by BMJ.

\section{A) Check for updates}

To cite Pisetsky DS, Spencer DM, Rovin B, et al. Ann Rheum Dis Epub ahead of print: [please include Day Month Year]. doi:10.1136/annrheumdis-2019-216259

Received 3 September 2019

Accepted 6 September 2019

Ann Rheum Dis 2019;0:1-2. doi:10.1136/annrheumdis-2019-216259

\section{REFERENCES}

1 Aringer M, Costenbader K, Daikh D, et al. 2019 European League against Rheumatism/American College of rheumatology classification criteria for systemic lupus erythematosus. Ann Rheum Dis 2019;78:1151-9.

2 Aringer M, Costenbader K, Daikh D, et al. 2019 European League against Rheumatism/American College of rheumatology classification criteria for systemic lupus erythematosus. Arthritis Rheumatol 2019.

3 Pisetsky DS, Spencer DM, Lipsky PE, et al. Assay variation in the detection of antinuclear antibodies in the sera of patients with established SLE. Ann Rheum Dis 2018:77:911-3.

4 Bizzaro N. Can solid-phase assays replace immunofluorescence for ANA screening? Ann Rheum Dis 2018:annrheumdis-2018-214805.

5 Infantino M, Manfredi M, Soda P, et al. ANA testing in 'real life'. Ann Rheum Dis 2018:annrheumdis-2018-214615.

6 Mahler M. Lack of standardisation of ANA and implications for drug development and precision medicine. Ann Rheum Dis 2018

7 Meroni PL, Chan EK, Damoiseaux J, et al. Unending story of the indirect immunofluorescence assay on HEp-2 cells: old problems and new solutions? Ann Rheum Dis 2018.

8 Pacheco Y, Monsalve DM, Acosta-Ampudia Y, et al. Antinuclear autoantibodies: discordance among four different assays. Ann Rheum Dis 2018:annrheumdis-2018-214693.
9 Pregnolato F, Borghi MO, Meroni PL. Pitfalls of antinuclear antibody detection in systemic lupus erythematosus: the positive experience of a national multicentre study. Ann Rheum Dis 2018.

10 Van Hoovels L, Schouwers S, Van den Bremt S, et al. Variation in antinuclear antibody detection by automated indirect immunofluorescence analysis. Ann Rheum Dis 2018.

11 Willems P DLE, Westhovens R, Vanderschueren S, et al. Antinuclear antibody as entry criteria for classification of systemic lupus erythematosus: pitfalls and opportunities. Ann Rheum Dis 2018.

12 Bossuyt X, Claessens J, De Langhe E, et al. Antinuclear antibodies by indirect immunofluorescence and solid phase assays. Ann Rheum Dis 2019:annrheumdis-2019-215443.

13 Tan EM, Cohen AS, Fries JF, et al. The 1982 revised criteria for the classification of systemic lupus erythematosus. Arthritis Rheum 1982;25:1271-7.

14 Petri M, Orbai A-M, Alarcón GS, et al. Derivation and validation of the systemic lupus international collaborating clinics classification criteria for systemic lupus erythematosus. Arthritis Rheum 2012:64:2677-86.

15 Pisetsky DS, Lipsky PE. Role of antinuclear antibody determinations in classification criteria for systemic lupus erythematosus: Comment on the article by Leuchten et al. Arthritis Care Res 2019;71.

16 Leuchten N, Hoyer A, Brinks R, et al. Performance of antinuclear antibodies for classifying systemic lupus erythematosus: a systematic literature review and metaregression of diagnostic data. Arthritis Care Res 2018;70:428-38.

17 Pisetsky DS, Thompson DK, Wajdula J, et al. Variability in antinuclear antibody testing to assess patient eligibility for clinical trials of novel treatments for systemic lupus erythematosus. Arthritis Rheumatol 2019;71:1534-8.

18 Wallace DJ, Stohl W, Furie RA, et al. A phase II, randomized, double-blind, placebocontrolled, dose-ranging study of belimumab in patients with active systemic lupus erythematosus. Arthritis Rheum 2009;61:1168-78.

19 Pisetsky DS. Antinuclear antibody testing - misunderstood or misbegotten? Nat Rev Rheumatol 2017;13:495-502.

20 Morais SA, Isenberg DA. A study of the influence of ethnicity on serology and clinical features in lupus. Lupus 2017;26:17-26.

21 Bossuyt $X$, Fieuws S. Detection of antinuclear antibodies: added value of solid phase assay? Ann Rheum Dis 2014;73:e10. 